\title{
Existence and location results for hinged beam equations with unbounded nonlinearities
}

\author{
J. Fialho ${ }^{\mathrm{a}, *}$, F. Minhós ${ }^{\mathrm{a}, \mathrm{b}}$ \\ a Centro de Investigação em Matemática e Aplicaçõ es da, Universidade de Évora (CIMA-UE), Portugal

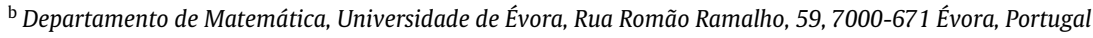

\section{A R T I C L E I N F O}

\section{Keywords:}

Ambrosetti-Prodi equations Lidstone boundary conditions Lower and upper solutions One-sided Nagumo condition Degree theory

\section{A B S T R A C T}

This work presents some existence, non-existence and location results for the problem composed by the fourth-order fully nonlinear equation

$$
u^{(4)}(x)+f\left(x, u(x), u^{\prime}(x), u^{\prime \prime}(x), u^{\prime \prime \prime}(x)\right)=\operatorname{sp}(x)
$$

for $x \in[0,1]$, where $f:[0,1] \times \mathbb{R}^{4} \rightarrow \mathbb{R}$ and $p:[0,1] \rightarrow \mathbb{R}^{+}$are continuous functions and $s$ is a real parameter, with the Lidstone boundary conditions

$$
u(0)=u(1)=u^{\prime \prime}(0)=u^{\prime \prime}(1)=0 .
$$

This problem models several phenomena, such as, the bending of an elastic beam simply supported at the endpoints.

The arguments used apply a lower and upper solutions technique, a priori estimations and topological degree theory. In this paper we replace the usual bilateral Nagumo condition by some one-sided conditions, which enables us to consider unbounded nonlinearities.

(c) 2009 Elsevier Ltd. All rights reserved.

\section{Introduction}

Fourth-order differential equations are often called beam equations due to their relevance in beam theory, namely in the study of the bending of an elastic beam. This paper considers the nonlinear full equation

$$
u^{(i v)}(x)+f\left(x, u(x), u^{\prime}(x), u^{\prime \prime}(x), u^{\prime \prime \prime}(x)\right)=s p(x)
$$

for $x \in[0,1]$, where $f:[0,1] \times \mathbb{R}^{4} \rightarrow \mathbb{R}$ and $p:[0,1] \rightarrow \mathbb{R}^{+}$are continuous functions and $s$ is a real parameter, with the boundary conditions

$$
u(0)=u(1)=u^{\prime \prime}(0)=u^{\prime \prime}(1)=0 .
$$

These types of condition, known as Lidstone boundary conditions, appear in several physical and engineering situations such as simply supported beams [1,2] and suspension bridges [3,4]. The related problems have been studied by many authors, either from a variational approach [5,6] or with topological techniques [7-10] or both [11]. Recently, some papers applied the lower and upper solutions method to more general boundary conditions such as nonlinear [12-14] and functional cases [15,16], some of them including the Lidstone case.

\footnotetext{
* Corresponding author.

E-mail addresses: jfzero@gmail.com (J. Fialho),fminhos@uevora.pt (F. Minhós).
} 
The bilateral Nagumo condition, used in some of the above papers, plays an important role to control the growth of the third derivative. In this work we apply a more general Nagumo-type assumption: a unilateral condition. Using this point of view, the results that exist in the literature for problem (1)-(2) $[17,18]$ are improved, because the nonlinearity can be unbounded from above or from below, following arguments suggested by [19,20].

It is pointed out that, for Lidstone problems, where there is no information about the third derivative on the boundary, the replacement of the bilateral condition by a unilateral one is not trivial. It requires a new a priori lemma and a new auxiliary problem in the proof of the main result.

The example contained in the final section illustrates this improvement and highlights some of the advantages of the lower and upper solutions in these boundary value problems, providing existence results, locating the solution and some derivatives, and adding some qualitative informations on them, for the values of the parameter $s$ such that there is a pair of lower and upper solutions of (1)-(2).

\section{Definitions and auxiliary results}

In this paper $C^{k}([0,1])$ denotes the space of real valued functions with continuous $i$-derivative in $[0,1]$, for $i=1, \ldots, k$, equipped with the norm

$$
\|y\|_{C^{k}}=\max _{0 \leq i \leq k}\left\{\left|y^{(i)}(x)\right|: x \in[0,1]\right\} .
$$

By $C([0,1])$ we denote the space of continuous functions with the norm $\|y\|=\max _{x \in[0,1]}|y(x)|$.

The one-sided Nagumo-type condition to be used and the consequent $a$ priori estimation are precise, as follows:

Definition 1. Given a subset $E \subset[0,1] \times \mathbb{R}^{4}$, a continuos function $f: E \rightarrow \mathbb{R}$ is said to satisfy the one-sided Nagumo-type condition in $E$ if there exists a real continuous function $h_{E}: \mathbb{R}_{0}^{+} \rightarrow[k,+\infty[$, for some $k>0$, such that

$$
f\left(x, y_{0}, y_{1}, y_{2}, y_{3}\right) \leq h_{E}\left(\left|y_{3}\right|\right), \quad \forall\left(x, y_{0}, y_{1}, y_{2}, y_{3}\right) \in E
$$

or

$$
f\left(x, y_{0}, y_{1}, y_{2}, y_{3}\right) \geq-h_{E}\left(\left|y_{3}\right|\right), \quad \forall\left(x, y_{0}, y_{1}, y_{2}, y_{3}\right) \in E,
$$

with

$$
\int_{0}^{+\infty} \frac{t}{h_{E}(t)} \mathrm{d} t=+\infty
$$

Lemma 2. Let $f:[0,1] \times \mathbb{R}^{4} \rightarrow \mathbb{R}$ be a continuous function, verifying Nagumo-type conditions (3) and (5) in

$$
E=\left\{\left(x, y_{0}, y_{1}, y_{2}, y_{3}\right) \in[0,1] \times \mathbb{R}^{4}: \gamma_{i}(x) \leq y_{i} \leq \Gamma_{i}(x), i=0,1,2\right\},
$$

where $\gamma_{i}(x)$ and $\Gamma_{i}(x)$ are continuous functions such that, for $i=0,1,2, \gamma_{i}(x) \leq \Gamma_{i}(x)$, for every $x \in[0,1]$.

Then for every $\rho>0$ there is $R>0$ such that every solution $u(x)$ of Eq. (1) verifying

$$
u^{\prime \prime \prime}(0) \geq-\rho, \quad u^{\prime \prime \prime}(1) \leq \rho
$$

and

$$
\gamma_{i}(x) \leq u^{(i)}(x) \leq \Gamma_{i}(x), \quad \forall x \in[0,1],
$$

for $i=0,1,2$, satisfies $\left\|u^{\prime \prime \prime}\right\|<R$.

Proof. Consider $u$, a solution of the Eq. (1) that satisfies (6) and (7), and define the non-negative real number $r:=$ $\max \left\{\Gamma_{2}(1)-\gamma_{2}(0), \Gamma_{2}(0)-\gamma_{2}(1)\right\}$.

Suppose $\rho>0$ be large enough such that for every $u$ solution of (1) we have $\left|u^{\prime \prime \prime}(x)\right| \leq \rho$, for every $x \in[0,1]$, and $\rho \geq r$. If $\rho=R$ then the proof is concluded.

Consider now that there is $u$, a solution of ( 1$)$ and $x_{0} \in[0,1]$, such that $\left|u^{\prime \prime \prime}\left(x_{0}\right)\right|>\rho$. If $\left|u^{\prime \prime \prime}(x)\right|>\rho$, for every $x \in[0,1]$, then, for $u^{\prime \prime \prime}(x)>\rho$, we obtain the following contradiction:

$$
\begin{aligned}
\Gamma_{2}(1)-\gamma_{2}(0) & \geq u^{\prime \prime}(1)-u^{\prime \prime}(0)=\int_{0}^{1} u^{\prime \prime \prime}(\tau) \mathrm{d} \tau \\
& >\int_{0}^{1} \rho \mathrm{d} \tau \geq \int_{0}^{1} r \mathrm{~d} \tau \geq \Gamma_{2}(1)-\gamma_{2}(0) .
\end{aligned}
$$

The case $u^{\prime \prime \prime}(x) \geq-\rho$, for every $x \in[0,1]$, follows similar arguments. So there is $x \in[0,1]$ such that $\left|u^{\prime \prime \prime}(x)\right| \leq \rho$. 
As the integrals $\int_{0}^{+\infty} \frac{t}{h_{E}(t)} \mathrm{d} t$ and $\int_{0}^{+\infty} \frac{\tau}{h_{E}(\tau)+|s| \mid p \|} \mathrm{d} \tau$ are of the same type, by (5), take $R_{1}>\rho$ such that

$$
\int_{\rho}^{R_{1}} \frac{\tau}{h_{E}(\tau)+|s|\|p\|} \mathrm{d} \tau>\max _{x \in[0,1]} \Gamma_{2}(x)-\min _{x \in[0,1]} \gamma_{2}(x) .
$$

Consider $x_{1} \in\left[0,1\left[\right.\right.$ such that $u^{\prime \prime \prime}\left(x_{1}\right)<-\rho$ or $\left.\left.x_{1} \in\right] 0,1\right]$ such that $u^{\prime \prime \prime}\left(x_{1}\right)>\rho$. In the first case take $\hat{x}_{1}$ such that $0 \leq \hat{x}_{1}<x_{1}$ and, for every $x \in\left[\hat{x}_{1}, x_{1}[\right.$,

$$
u^{\prime \prime \prime}\left(\hat{x}_{1}\right)=-\rho \text { and } u^{\prime \prime \prime}(x)<-\rho \text {. }
$$

By an adequate change of variable and (8), we obtain

$$
\begin{aligned}
\int_{-u^{\prime \prime \prime}\left(\widehat{x}_{1}\right)}^{-u^{\prime \prime \prime}\left(x_{1}\right)} \frac{\tau}{h_{E}(\tau)+|s|\|p\|} \mathrm{d} \tau & =\int_{\widehat{x}_{1}}^{x_{1}} \frac{-u^{\prime \prime \prime}(x)}{h_{E}\left(-u^{\prime \prime \prime}(x)\right)+|s|\|p\|} \cdot\left(-u^{(i v)}(x)\right) \mathrm{d} x \\
& =\int_{\widehat{x}_{1}}^{x_{1}} \frac{f\left(x, u, u^{\prime}, u^{\prime \prime}, u^{\prime \prime \prime}\right)-s p(x)}{h_{E}\left(-u^{\prime \prime \prime}(x)\right)+|s|\|p\|}\left(-u^{\prime \prime \prime}(x)\right) \mathrm{d} x \\
& \leq \int_{\widehat{x}_{1}}^{x_{1}}-u^{\prime \prime \prime}(x) \mathrm{d} x=u^{\prime \prime}\left(\widehat{x}_{1}\right)-u^{\prime \prime}\left(x_{1}\right) \\
& \leq \max _{x \in[0,1]} \Gamma_{2}(x)-\min _{x \in[0,1]} \gamma_{2}(x) \\
& <\int_{\rho}^{R_{1}} \frac{\tau}{h_{E}(\tau)+|s|\|p\|} \mathrm{d} \tau,
\end{aligned}
$$

and therefore that $u^{\prime \prime \prime}\left(x_{1}\right)>-R_{1}$. By the arbitrariness of $x_{1}$, then for every $x \in\left[0,1\right.$ [ such that $u^{\prime \prime \prime}(x)<-\rho$ the inequality $u^{\prime \prime \prime}(x)>-R_{1}$ holds. In a similar way it can be proved that $u^{\prime \prime \prime}\left(x_{1}\right)<R_{1}$, and so $\left|u^{\prime \prime \prime}(x)\right| \leq R_{1}$, for every $x \in[0,1]$.

Consider now $\rho<r$, and take $R_{2}>r$ such that

$$
\int_{r}^{R_{2}} \frac{\tau}{h_{E}(\tau)+|s|\|p\|} \mathrm{d} \tau>\max _{x \in[0,1]} \Gamma_{2}(x)-\min _{x \in[0,1]} \gamma_{2}(x) .
$$

By (6), there is $x \in[0,1]$ such that $\left|u^{\prime \prime \prime}(x)\right| \leq r$. If $\left|u^{\prime \prime \prime}(x)\right| \leq r$ holds for every $x \in[0,1]$, then the proof is concluded. Otherwise, we take $x_{2} \in\left[0,1\left[\right.\right.$ such that $u^{\prime \prime \prime}\left(\bar{x}_{2}\right)<-r$ or $\left.\left.x_{2} \in\right] 0,1\right]$ such that $u^{\prime \prime \prime}\left(x_{2}\right)>r$. In the first case consider $0 \leq \hat{x}_{2} \leq x_{2}$ with

$$
u^{\prime \prime \prime}\left(\hat{x}_{2}\right)=-r \quad \text { and } \quad u^{\prime \prime \prime}(x)<-r, \quad \forall x \in\left[\hat{x}_{2}, x_{2}[\right.
$$

Applying a similar method as in (9), we obtain

$$
\int_{-u^{\prime \prime \prime}\left(\widehat{x}_{2}\right)}^{-u^{\prime \prime \prime}\left(x_{2}\right)} \frac{\tau}{h_{E}(\tau)+|s|\|p\|} \mathrm{d} \tau<\int_{r}^{R_{2}} \frac{\tau}{h_{E}(\tau)+|s|\|p\|} \mathrm{d} \tau
$$

and so $u^{\prime \prime \prime}\left(x_{2}\right)>-R_{2}$. Arguing as above it can be shown that when $u^{\prime \prime \prime}\left(x_{2}\right)>r$ the inequality $u^{\prime \prime \prime}\left(x_{2}\right)<R_{2}$ still holds. Therefore $\left|u^{\prime \prime \prime}(x)\right| \leq R_{1}$, for every $x \in[0,1]$.

Taking $R=\max \left\{R_{1}, R_{2}\right\}$ then $\left|u^{\prime \prime \prime}(x)\right| \leq R$, for every $x \in[0,1]$.

Remark 3. If the function $f$ verifies (4), the previous estimate still holds replacing, in Lemma 2, (6) by

$$
u^{\prime \prime \prime}(0) \leq \rho, \quad u^{\prime \prime \prime}(1) \geq-\rho .
$$

Remark 4. Observe that $R$ depends only on the functions $h_{E}, \gamma_{2}$ and $\Gamma_{2}$, and not on the boundary conditions. Moreover, if $s$ belongs to a bounded set, then $R$ can be considered the same, independently of $s$.

The functions used as upper and lower solutions are defined as a pair:

Definition 5. The functions $\alpha, \beta \in C^{4}(] 0,1[) \cap C^{2}([0,1])$ verifying

$$
\alpha^{(i)}(x) \leq \beta^{(i)}(x), \quad i=0,1,2, \forall x \in[0,1],
$$

define a pair of lower and upper solutions of problem (1)-(2) if the following conditions are satisfied:

(i)

$$
\begin{aligned}
& \alpha^{(i v)}(x)+f\left(x, \alpha(x), \alpha^{\prime}(x), \alpha^{\prime \prime}(x), \alpha^{\prime \prime \prime}(x)\right) \geq s p(x) \\
& \beta^{(i v)}(x)+f\left(x, \beta(x), \beta^{\prime}(x), \beta^{\prime \prime}(x), \beta^{\prime \prime \prime}(x)\right) \leq s p(x)
\end{aligned}
$$


(ii)

$$
\begin{array}{ll}
\alpha(0) \leq 0, & \alpha^{\prime \prime}(0) \leq 0, \quad \alpha^{\prime \prime}(1) \leq 0 \\
\beta(0) \geq 0, & \beta^{\prime \prime}(0) \geq 0, \quad \beta^{\prime \prime}(1) \geq 0
\end{array}
$$

$$
\alpha^{\prime}(0)-\beta^{\prime}(0) \leq \min \{\beta(0)-\beta(1), \alpha(1)-\alpha(0)\}
$$

As was shown in [17], condition (iii) cannot be removed for this type of definition. However, if the minimum in (iii) is non-positive then assumption (12) can be replaced by $\alpha^{\prime \prime}(x) \leq \beta^{\prime \prime}(x)$, for every $x \in[0,1]$, as the other inequalities are obtained from integration.

\section{Existence and location result}

For values of the parameter s such that there are lower and upper solutions of (1)-(2), we can be obtain the following existence and location result, where the nonlinear part can be unbounded from above or from below.

Theorem 6. Suppose that there is a pair of upper and lower solutions of the problem (1)-(2), $\alpha(x)$ and $\beta(x)$, respectively. Let $f:[0,1] \times \mathbb{R}^{4} \rightarrow \mathbb{R}$ be a continuous function satisfying the one-sided Nagumo conditions (3), or (4) and (5) in

$$
E_{*}=\left\{\begin{array}{c}
\left(x, y_{0}, y_{1}, y_{2}, y_{3}\right) \in[0,1] \times \mathbb{R}^{4}: \alpha(x) \leq y_{0} \leq \beta(x), \\
\alpha^{\prime}(x) \leq y_{1} \leq \beta^{\prime}(x), \alpha^{\prime \prime}(x) \leq y_{2} \leq \beta^{\prime \prime}(x)
\end{array}\right\}
$$

and

$$
f\left(x, \alpha, \alpha^{\prime}, y_{2}, y_{3}\right) \leq f\left(x, y_{0}, y_{1}, y_{2}, y_{3}\right) \leq f\left(x, \beta, \beta^{\prime}, y_{2}, y_{3}\right),
$$

for $\alpha(x) \leq y_{0} \leq \beta(x), \alpha^{\prime}(x) \leq y_{1} \leq \beta^{\prime}(x)$ and for fixed $\left(x, y_{2}, y_{3}\right) \in[0,1] \times \mathbb{R}^{2}$. Then the problem (1)-(2) has at least a solution $u(x) \in C^{4}([0,1])$, satisfying

$$
\alpha^{(i)}(x) \leq u^{(i)}(x) \leq \beta^{(i)}(x), \quad \text { for } i=0,1,2, \forall x \in[0,1] .
$$

Proof. Consider the continuous truncations $\delta_{i}$ given by

$$
\delta_{i}\left(x, y_{i}\right)= \begin{cases}\alpha^{(i)}(x) & \text { if } y_{i}<\alpha^{(i)}(x) \\ y_{i} & \text { if } \alpha^{(i)}(x) \leq y_{i} \leq \beta^{(i)}(x), \quad i=0,1,2 . \\ \beta^{(i)}(x) & \text { if } y^{(i)}>\beta^{(i)}(x)\end{cases}
$$

For $\lambda \in[0,1]$, consider the homotopic equation

$$
u^{(i v)}(x)=\lambda\left[\operatorname{sp}(x)-f\left(x, \delta_{0}(x, u), \delta_{1}\left(x, u^{\prime}\right), \delta_{2}\left(x, u^{\prime \prime}\right), u^{\prime \prime \prime}\right)\right]+u^{\prime \prime}(x)-\lambda \delta_{2}\left(x, u^{\prime \prime}\right),
$$

and the boundary conditions

$$
\begin{aligned}
& u(0)=0, \quad u(1)=0, \\
& u^{\prime \prime \prime}(0)=\lambda\left[u^{\prime \prime \prime}(0)+u^{\prime \prime}(0)\right], \\
& u^{\prime \prime \prime}(1)=\lambda\left[u^{\prime \prime \prime}(1)-u^{\prime \prime}(1)\right] .
\end{aligned}
$$

Let $r_{2}>0$ be large enough, such that, for every $x \in[0,1]$,

$$
\begin{aligned}
& -r_{2}<\alpha^{\prime \prime}(x) \leq \beta^{\prime \prime}(x)<r_{2}, \\
& s p(x)-f\left(x, \beta(x), \beta^{\prime}(x), \beta^{\prime \prime}(x), 0\right)+r_{2}-\beta^{\prime \prime}(x)>0, \\
& s p(x)-f\left(x, \alpha(x), \alpha^{\prime}(x), \alpha^{\prime \prime}(x), 0\right)-r_{2}-\alpha^{\prime \prime}(x)<0,
\end{aligned}
$$

and, for every $u$ solution of (15) and (16)

$$
\left|u^{\prime \prime \prime}(0)\right| \leq r_{2}, \quad\left|u^{\prime \prime \prime}(1)\right| \leq r_{2} .
$$

Step 1- For every solution $u(x)$ of the problem (15)-(16) we have

$$
\left|u^{\prime \prime}(x)\right|<r_{2},\left|u^{\prime}(x)\right|<r_{1},|u(x)|<r_{0}, \quad \forall x \in[0,1],
$$

with $r_{1}:=r_{2}+u^{\prime}(0)$ and $r_{0}>r_{1}$, independently of $\lambda \in[0,1]$.

Let $u$ be a solution of (15) and (16). By contradiction assume that there are $\lambda \in[0,1]$ and $x \in[0,1]$ such that $\left|u^{\prime \prime}(x)\right| \geq r_{2}$. In the case $u^{\prime \prime}(x) \geq r_{2}$ define

$$
\max _{x \in[0,1]} u^{\prime \prime}(x):=u^{\prime \prime}\left(x_{0}\right) \geq r_{2}>0
$$


If $\left.x_{0} \in\right] 0,1\left[\right.$ then $u^{\prime \prime \prime}\left(x_{0}\right)=0$ and $u^{(i v)}\left(x_{0}\right) \leq 0$. Therefore, by (13) and (18), for $\lambda \in[0,1]$ we obtain the following contradiction:

$$
\begin{aligned}
0 & \geq u^{(i v)}\left(x_{0}\right) \\
& =\lambda\left[\operatorname{sp}\left(x_{0}\right)-f\left(x_{0}, \delta_{0}\left(x_{0}, u\right), \delta_{1}\left(x_{0}, u^{\prime}\right), \beta^{\prime \prime}\left(x_{0}\right), 0\right)\right]+u^{\prime \prime}\left(x_{0}\right)-\beta^{\prime \prime}\left(x_{0}\right) \\
& \geq \lambda\left[\operatorname{sp}\left(x_{0}\right)-f\left(x_{0}, \beta\left(x_{0}\right), \beta^{\prime}\left(x_{0}\right), \beta^{\prime \prime}\left(x_{0}\right), 0\right)\right]+r_{2}-\beta^{\prime \prime}\left(x_{0}\right)>0 . \\
\text { If } x_{0} & =0, \text { for } \lambda \in] 0,1], \text { by }(19) \text { the contradiction is } \\
0 & \geq u^{\prime \prime \prime}(0)=\lambda\left[u^{\prime \prime \prime}(0)+u^{\prime \prime}(0)\right] \geq \lambda\left(u^{\prime \prime \prime}(0)+r_{2}\right)>0 .
\end{aligned}
$$

For $\lambda=0$, by (16), $u^{\prime \prime \prime}(0)=0$ and $0 \geq u^{(i v)}(0)=u^{\prime \prime}(0) \geq r_{2}>0$. The situation is analogous for $x_{0}=1$, and, therefore, $u^{\prime \prime}(x)<r_{2}$, for every $x \in[0,1]$. The case $u^{\prime \prime}(x) \leq-r_{2}$ is similarly analogous, and so

$$
\left|u^{\prime \prime}(x)\right|<r_{2}, \quad \forall x \in[0,1], \forall \lambda \in[0,1] .
$$

Integrating in $[0, x], u^{\prime}(x)-u^{\prime}(0)=\int_{0}^{x} u^{\prime \prime}(s) \mathrm{d} s<r_{2}$, and

$$
\left|u^{\prime}(x)\right|<r_{2}+u^{\prime}(0), \quad \forall x \in[0,1], \forall \lambda \in[0,1] .
$$

By integration, $u(x)-u(0)=\int_{0}^{x} u^{\prime}(s) \mathrm{d} s \leq \int_{0}^{x} r_{1} \mathrm{~d} s \leq r_{1}<r_{0}$.

With the same arguments it can be proved that $u(x)>-r_{0}$ and

$$
|u(x)|<r_{0}, \forall x \in[0,1] .
$$

Step 2- There is $R>0$, such that every solution $u(x)$ of the problem (15)-(16) verifies

$$
\left|u^{\prime \prime \prime}(x)\right|<R, \quad \forall x \in[0,1],
$$

independently of $\lambda \in[0,1]$.

In order to apply the Lemma 2, define the set

$$
E_{r}=\left\{\left(x, y_{0}, y_{1}, y_{2}, y_{3}\right) \in[0,1] \times \mathbb{R}^{4}:-r_{1} \leq y_{i} \leq r_{1}, i=0,1,-r_{2} \leq y_{2} \leq r_{2}\right\},
$$

with $r_{1}, r_{2}$ given by Step 1 , and, for $\lambda \in[0,1]$, the function $F_{\lambda}: E_{r} \rightarrow \mathbb{R}$ defined by

$$
F_{\lambda}\left(x, y_{0}, y_{1}, y_{2}, y_{3}\right)=\lambda f\left(x, \delta_{0}\left(x, y_{0}\right), \delta_{1}\left(x, y_{1}\right), \delta_{2}\left(x, y_{2}\right), y_{3}\right)+y_{2}-\lambda \delta_{2}\left(x, y_{2}\right) \text {. }
$$

If $f$ verifies (3) in $E_{r}$, then

$$
F_{\lambda}\left(x, y_{0}, y_{1}, y_{2}, y_{3}\right) \leq \lambda h_{E_{r}}\left(\left|y_{3}\right|\right)+r_{2}-\lambda \alpha^{\prime \prime}(x) \leq h_{E_{r}}\left(\left|y_{3}\right|\right)+2 r_{2},
$$

and $F_{\lambda}$ satisfies (3) with $h_{E}$ replaced by $\bar{h}_{E_{r}}(x)=h_{E_{r}}(x)+2 r_{2}$ in $E_{r}$.

If condition (4) holds in $E_{r}$, we will obtain, in a similar way,

$$
F_{\lambda}\left(x, y_{0}, y_{1}, y_{2}, y_{3}\right) \geq-\lambda h_{E_{r}}\left(\left|y_{3}\right|\right)-r_{2}-\lambda \beta^{\prime \prime}(x) \geq-\left(h_{E_{r}}\left(\left|y_{3}\right|\right)+2 r_{2}\right) .
$$

Condition (5) holds as

$$
\begin{aligned}
\int_{0}^{+\infty} \frac{t}{\bar{h}_{E_{r}}(t)} \mathrm{d} t & =\int_{0}^{+\infty} \frac{t}{h_{E_{r}}(t)+2 r_{2}} \mathrm{~d} t \\
& \geq \frac{1}{1+\frac{2 r_{2}}{k}} \int_{0}^{+\infty} \frac{t}{h_{E_{r}}(t)} \mathrm{d} t=+\infty
\end{aligned}
$$

By (19), Lemma 2 holds with $\gamma_{i}(x)=-r_{1}, \Gamma_{i}(x)=r_{1}, i=0,1, \gamma_{2}(x)=-r_{2}, \Gamma_{2}(x)=r_{2}$ and $\rho=r_{2}$. Therefore, there is $R>0$ such that

$$
\left|u^{\prime \prime \prime}(x)\right|<R, \quad \forall x \in[0,1] .
$$

Observe that as $r_{2}$ and $h_{E r}$ do not depend on $\lambda$ then $R$ does not depend on $\lambda$.

Step 3- Problem (15)-(16) has at least a solution $u_{1}(x)$ for $\lambda=1$.

Define the operators $\mathcal{L}: C^{4}([0,1]) \subset C^{3}([0,1]) \rightarrow C([0,1]) \times \mathbb{R}^{4}$ given by

$$
\mathcal{L} u=\left(u^{(i v)}-u^{\prime \prime}, u(0), u(1), u^{\prime \prime \prime}(0), u^{\prime \prime \prime}(1)\right)
$$

and $\mathcal{N}_{\lambda}: C^{3}([0,1]) \rightarrow C([0,1]) \times \mathbb{R}^{4}$ by

$$
\mathcal{N}_{\lambda}=\left(\begin{array}{c}
\lambda\left[\operatorname{sp}(x)-f\left(x, \delta_{0}(x, u), \delta_{1}\left(x, u^{\prime}\right), \delta_{2}\left(x, u^{\prime \prime}\right), u^{\prime \prime \prime}(x)\right)\right]-\lambda \delta_{2}\left(x, u^{\prime \prime}\right), \\
0,0, \lambda\left[u^{\prime \prime \prime}(0)+u^{\prime \prime}(0)\right], \lambda\left[u^{\prime \prime \prime}(1)-u^{\prime \prime}(1)\right]
\end{array}\right) .
$$


As $\mathcal{L}^{-1}$ is compact then we can define the completely continuous operator $\mathcal{T}_{\lambda}:\left(C^{4}([0,1]), \mathbb{R}\right) \rightarrow\left(C^{4}([0,1]), \mathbb{R}\right)$ given by $\mathcal{T}_{\lambda}(u)=\mathcal{L}^{-1} \mathcal{N}_{\lambda}(u)$.

For $r_{1}, r_{2}$ and $R$ given by Steps 1 and 2 , consider the set

$$
\Omega=\left\{y \in C^{3}([0,1]):\left\|y^{(i)}\right\|<r_{1}, i=0,1,\left\|y^{\prime \prime}\right\|<r_{2},\left\|y^{\prime \prime \prime}\right\|<R\right\} .
$$

Therefore, the degree $d\left(\mathcal{T}_{\lambda}, \Omega, 0\right)$ is well defined for every $\lambda \in[0,1]$, and by the invariance under homotopy, $d\left(\mathcal{T}_{0}, \Omega, 0\right)=$ $d\left(\mathcal{T}_{1}, \Omega, 0\right)$.

The equation $T_{0}(u)=u$ is equivalent to the homogeneous problem

$$
\left\{\begin{array}{l}
u^{(i v)}(x)-u^{\prime \prime}(x)=0 \\
u(0)=u(1)=u^{\prime \prime \prime}(1)=u^{\prime \prime \prime}(0)=0
\end{array}\right.
$$

that admits only a trivial solution. Then, by degree theory, $d\left(\mathcal{T}_{0}, \Omega, 0\right)= \pm 1$, and the equation $u=\mathcal{T}_{1}(u)$ has at least a solution. That is, the problem composed by the equation

$$
u^{(i v)}(x)=s p(x)-f\left(x, \delta_{0}(x, u), \delta_{1}\left(x, u^{\prime}\right), \delta_{2}\left(x, u^{\prime \prime}\right), u^{\prime \prime \prime}(x)\right)+u^{\prime \prime}(x)-\delta_{2}\left(x, u^{\prime \prime}\right)
$$

with the initial boundary conditions (2) has at least one solution $u_{1}(x)$ in $\Omega$.

Step 4- The function $u_{1}(x)$ is a solution of the problem (1)-(2)

The function $u_{1}(x)$ will be a solution of the initial problem (1)-(2) if it verifies $\alpha^{(i)}(x) \leq u_{1}^{(i)}(x) \leq \beta^{(i)}(x), i=0,1,2$, $\forall x \in[0,1]$.

Suppose, by contradiction, that there is $x \in[0,1]$ such that $\alpha^{\prime \prime}(x)>u_{1}^{\prime \prime}(x)$, and define

$$
\min _{x \in[0,1]}\left[u_{1}^{\prime \prime}(x)-\alpha^{\prime \prime}(x)\right]:=u_{1}^{\prime \prime}\left(x_{1}\right)-\alpha^{\prime \prime}\left(x_{1}\right)<0 .
$$

If $\left.x_{1} \in\right] 0,1\left[\right.$, then $u_{1}^{\prime \prime \prime}\left(x_{1}\right)=\alpha^{\prime \prime \prime}\left(x_{1}\right)$ and $u^{(i v)}\left(x_{1}\right)-\alpha^{(i v)}\left(x_{1}\right) \geq 0$.

By Definition 5 and (13) we obtain the contradiction

$$
\begin{aligned}
\alpha^{(i v)}\left(x_{1}\right) & \leq u_{1}^{(i v)}\left(x_{1}\right) \\
& =s p\left(x_{1}\right)-f\left(x_{1}, \delta_{0}\left(x_{1}, u\right), \delta_{1}\left(x_{1}, u^{\prime}\right), \alpha^{\prime \prime}\left(x_{1}\right), \alpha^{\prime \prime \prime}\left(x_{1}\right)\right)+u^{\prime \prime}\left(x_{1}\right)-\alpha^{\prime \prime}\left(x_{1}\right) \\
& <s p\left(x_{1}\right)-f\left(x_{1}, \alpha\left(x_{1}\right), \alpha^{\prime}\left(x_{1}\right), \alpha^{\prime \prime}\left(x_{1}\right), \alpha^{\prime \prime \prime}\left(x_{1}\right)\right) \leq \alpha^{(i v)}\left(x_{1}\right) .
\end{aligned}
$$

If $x_{1}=0$ or $x_{1}=1$ the contradiction is trivial, by Definition 5 (ii).

Therefore $\alpha^{\prime \prime}(x) \leq u_{1}^{\prime \prime}(x)$, for every $x \in[0,1]$. In a similar way it can be proved that $u_{1}^{\prime \prime}(x) \leq \beta^{\prime \prime}(x)$, and so $\alpha^{\prime \prime}(x) \leq u_{1}^{\prime \prime}(x) \leq \beta^{\prime \prime}(x)$, for every $x \in[0,1]$

As, by (2),

$$
\begin{aligned}
0 & =\int_{0}^{1} u_{1}^{\prime}(x) \mathrm{d} x=\int_{0}^{1}\left(u_{1}^{\prime}(0)+\int_{0}^{x} u_{1}^{\prime \prime}(s) \mathrm{d} s\right) \mathrm{d} x \\
& =u_{1}^{\prime}(0)+\int_{0}^{1} \int_{0}^{x} u_{1}^{\prime \prime}(s) \mathrm{d} s \mathrm{~d} x,
\end{aligned}
$$

then $u_{1}^{\prime}(0)=-\int_{0}^{1} \int_{0}^{x} u_{1}^{\prime \prime}(s) \mathrm{d} s \mathrm{~d} x$. By this technique

$$
\int_{0}^{1} \int_{0}^{x} \alpha^{\prime \prime}(s) \mathrm{d} s \mathrm{~d} x=\alpha(1)-\alpha(0)-\alpha^{\prime}(0),
$$

and, by Definition 5(iii) and (17),

$$
\begin{aligned}
-\beta^{\prime}(0) & \leq \alpha(1)-\alpha(0)-\alpha^{\prime}(0)=\int_{0}^{1} \int_{0}^{x} \alpha^{\prime \prime}(s) \mathrm{d} s \mathrm{~d} x \\
& \leq \int_{0}^{1} \int_{0}^{x} u_{1}^{\prime \prime}(s) \mathrm{d} s \mathrm{~d} x=-u_{1}^{\prime}(0) .
\end{aligned}
$$

Therefore, $u_{1}^{\prime}(0) \leq \beta^{\prime}(0)$ and, by integration of (17), one obtains

$$
u_{1}^{\prime}(x)-u_{1}^{\prime}(0)=\int_{0}^{x} u_{1}^{\prime \prime}(s) \mathrm{d} s \leq \int_{0}^{x} \beta^{\prime \prime}(s) \mathrm{d} s=\beta^{\prime}(x)-\beta^{\prime}(0)
$$

and

$$
u_{1}^{\prime}(x) \leq \beta^{\prime}(x)-\beta^{\prime}(0)+u_{1}^{\prime}(0) \leq \beta^{\prime}(x), \quad \forall x \in[0,1] .
$$


The relation $\alpha^{\prime}(x) \leq u_{1}^{\prime}(x)$, for every $x \in[0,1]$, can be proved by similar arguments. Then $\alpha^{\prime}(x) \leq u_{1}^{\prime}(x) \leq \beta^{\prime}(x)$, for every $x \in[0,1]$. By Definition 5 (ii)

$$
\begin{aligned}
\alpha(x) & \leq \int_{0}^{x} \alpha^{\prime}(s) \mathrm{d} s \leq \int_{0}^{x} u_{1}^{\prime}(s) \mathrm{d} s=u_{1}(x) \\
& \leq \int_{0}^{x} \beta^{\prime}(s) \mathrm{d} s=\beta(x)-\beta(0) \leq \beta(x) .
\end{aligned}
$$

Therefore $u_{1}(x)$ is a solution for problem (1)-(2).

\section{Example}

Consider, for $k \in \mathbb{N}_{0}$, the fourth-order equation

$$
u^{(i v)}(x)+e^{u(x)}+\arctan \left(u^{\prime}(x)\right)-u^{\prime \prime}(x)^{3}-\left[u^{\prime \prime \prime}(x)\right]^{2 k+4}=s p(x) .
$$

The functions $\alpha$ and $\beta$ given by $\alpha:=-x^{2}-1$ and $\beta:=x+1$ are, respectively, upper and lower solutions of the problem (20)-(2), for values of $s$ such that

$$
K_{0}:=\frac{\mathrm{e}^{2}+\frac{\pi}{4}}{\min _{x \in[0,1]} p(x)} \leq s \leq \frac{\mathrm{e}^{-2}-\frac{\pi}{2}+8}{\max _{x \in[0,1]} p(x)}:=K_{1} .
$$

Defining

$$
E^{\prime}=\left\{\begin{array}{c}
\left(x, y_{0}, y_{1}, y_{2}, y_{3}\right) \in[0,1] \times \mathbb{R}^{4}:-x^{2}-1 \leq y_{0} \leq x+1, \\
-2 x \leq y_{1} \leq 1,-2 \leq y_{2} \leq 0
\end{array}\right\},
$$

the continuous function $f: E^{\prime} \rightarrow \mathbb{R}$, given by

$$
f\left(x, y_{0}, y_{1}, y_{2}, y_{3}\right)=\exp \left(y_{0}\right)+\arctan \left(y_{1}\right)-y_{2}^{3}-y_{3}^{2 k+4}, k \in \mathbb{N}_{0},
$$

verifies the Nagumo condition (3) and assumption (13), with $h_{E^{\prime}}\left(y_{3}\right)=e^{2}+\frac{\pi}{2}$. Then, by Theorem 6, there is a solution $u(x)$ of problem (20)-(2) such that

$$
-x^{2}-1 \leq u(x) \leq x+1,-2 x \leq u^{\prime}(x) \leq 1,-2 \leq u^{\prime \prime}(x) \leq 0, \quad \forall x \in[0,1] .
$$

Notice that the nonlinearity $f$ given by (21) does not verify the two-sided Nagumo type conditions and, therefore, [17] cannot be applied to (2) and (20). In fact, suppose by contradiction that there are a set $E$ and a positive function $\varphi$ such that $\left|f\left(x, y_{0}, y_{1}, y_{2}, y_{3}\right)\right| \leq \varphi\left(\left|y_{3}\right|\right)$ in $E$ and

$$
\int_{0}^{+\infty} \frac{s}{\varphi(s)}=+\infty
$$

Consider, in particular, that

$$
f\left(x, y_{0}, y_{1}, y_{2}, y_{3}\right) \leq \varphi\left(\left|y_{3}\right|\right), \quad \forall\left(x, y_{0}, y_{1}, y_{2}, y_{3}\right) \in E
$$

and $\left(0,0,0, y_{3}\right) \in E$. So, for $x \in[0,1], y_{0}=0, y_{1}=0, y_{2}=0$ and $y_{3} \in \mathbb{R}^{+}$,

$$
f\left(x, 0,0,0, y_{3}\right)=1+y_{3}^{2 k+4} \leq \varphi\left(\left|y_{3}\right|\right) .
$$

As $\int_{0}^{+\infty} \frac{s}{1+s^{2 k+4}} \mathrm{~d} s, k \in \mathbb{N}_{0}$, is finite, then the following contradiction is obtained:

$$
+\infty>\int_{0}^{+\infty} \frac{s}{1+s^{2 k+4}} \mathrm{~d} s \geq \int_{0}^{+\infty} \frac{s}{\varphi(s)} \mathrm{d} s=+\infty .
$$

\section{References}

[1] C.P. Gupta, Existence and uniqueness theorems for the bending of an elastic beam equation, Appl. Anal. 26 (1988) 289-304.

[2] C.P. Gupta, Existence and uniqueness theorems for a fourth order boundary value problem of Sturm-Liouville type, Differential Integral Equations 4 (2) (1991) 397-410.

[3] P. Drábek, G. Holubová, A. Matas, P. Nečessal, Nonlinear models of suspension bridges: Discussion of results, Appl. Math. 48 (2003) $497-514$.

[4] A.C. Lazer, P.J. Mckenna, Large-amplitude periodic oscillations in suspension bridges: Some new connections with nonlinear analysis, SIAM Rev. 32 (1990) 537-578.

[5] M.R. Grossinho, S. Tersian, The dual variational principle and equilibria for a beam resting on a discontinuous nonlinear elastic foundation, Nonlinear Anal. Series A: Theory Methods 41 (3-4) (2000) 417-431.

[6] T. Gyulov, S. Tersian, Existence of trivial and nontrivial solutions of a fourth-order ordinary differential equation, Electron. J. Differential Equations $2004(41)(2004) 1-14$ 
[7] Z. Bai, The method of lower and upper solutions for a bending of an elastic beam equation, J. Math. Anal. Appl. 248 (2000) 195-202.

[8] A. Cabada, The method of lower and upper solutions for second, third, fourth, and higher order boundary value problems, J. Math. Anal. Appl. 185 (2) (1994) 302-320.

[9] X.L. Lu, W.T. Li, Positive solutions of the non-linear fourth-order beam equation with three parameters, J. Math. Anal. Appl. 303 (2005) $150-163$.

[10] M. Ruyun, Z. Jihui, F. Shengmao, The method of lower and upper solutions for fourth-order boundary value problems, J. Math. Anal. Appl. 215 (1997) $415-422$.

[11] C. de Coster, Sanchez, Upper and lower solutions, Ambrosetti-Prodi problem and positive solutions for a fourth order O.D.E, Riv. Mat. Pura Appl. 14 (1994) 57-82.

[12] J. Ehme, P. Eloe, J. Henderson, Upper and lower solutions method for fully nonlinear boundary value problems, J. Differential Equations 180 (2002) 51-64.

[13] D. Franco, D. O'Regan, J. Perán, Fourth-order problems with nonlinear boundary conditions, J. Comput. Appl. Math. 174 (2005) 315-327.

[14] F. Minhós, T. Gyulov, A.I. Santos, On an elastic beam fully equation with nonlinear boundary conditions, in: R. Agarwal, K. Perera (Eds.), Proc. of Conference on Differential \& Difference Equations and Applications (2005), Hindawi Publishing Corporation, 2006, pp. 805-814.

[15] A. Cabada, F. Minhós, Fully nonlinear fourth order equations with functional boundary conditions, J. Math. Anal. Appl. 340 (2008) $239-251$.

[16] A. Cabada, R. Pouso, F. Minhós, Extremal solutions to fourth-order functional boundary value problems including multipoint condition, Nonlinear Anal. RWA, doi:10.1016/j.nonrwa.2008.03.026.

[17] F. Minhós, T. Gyulov, A.I. Santos, Existence and location result for a fourth order boundary value problem, Discrete Contin. Dyn. Syst., Supp. (2005) 662-671.

[18] F. Minhós, T. Gyulov, A.I. Santos, Existence and location results for the bending of an elastic beam, in: M. Fila, A. Handlovicova, K. Mikula, M. Medved, P. Quittner, D. Sevcovic (Eds.), Proceedings Equadiff 11, 2007, pp. 273-282.

[19] M.R. Grossinho, F.M. Minhós, A.I. Santos, Solvability of some third-order boundary value problems with asymmetric unbounded linearities, Nonlinear Anal. 62 (2005) $1235-1250$.

[20] M.R. Grossinho, F. Minhós, A.I. Santos, A note on a class of problems for a higher order fully nonlinear equation under one sided Nagumo type condition, Nonlinear Anal., doi:10.1016/j.na.2008.08.011. 